\title{
SEARCHING FOR TROJAN ASTEROIDS IN THE HD 209458 SYSTEM: SPACE-BASED MOST PHOTOMETRY AND DYNAMICAL MODELING
}

\author{
Reka Moldovan $^{1,4}$, Jaymie M. Matthews ${ }^{1}$, Brett Gladman ${ }^{1}$, William F. Bottke ${ }^{2}$, and David VokrouhlickÝ ${ }^{3}$ \\ ${ }^{1}$ Department of Physics and Astronomy, The University of British Columbia, 6224 Agricultural Road, Vancouver, BC V6T 1Z1, Canada; rmoldova@ eos.ubc.ca \\ ${ }^{2}$ Department of Space Studies, Southwest Research Institute, 1050 Walnut Street, Suite 400, Boulder, CO 80302, USA \\ ${ }^{3}$ Institute of Astronomy, Charles University, Prague, V Holešovičkách 2, 18000 Prague 8, Czech Republic \\ Received 2009 November 25; accepted 2010 April 19; published 2010 May 17
}

\begin{abstract}
We have searched Microvariability and Oscillations of Stars (MOST) satellite photometry obtained in 2004, 2005, and 2007 of the solar-type star HD 209458 for Trojan asteroid swarms dynamically coupled with the system's transiting "hot Jupiter" HD 209458b. Observations of the presence and nature of asteroids around other stars would provide unique constraints on migration models of exoplanetary systems. Our results set an upper limit on the optical depth of Trojans in the HD 209458 system that can be used to guide current and future searches of similar systems by upcoming missions. Using cross-correlation methods with artificial signals implanted in the data, we find that our detection limit corresponds to a relative Trojan transit depth of $1 \times 10^{-4}$, equivalent to $\sim 1$ lunar mass of asteroids, assuming power-law Trojan size distributions similar to Jupiter's Trojans in our solar system. We confirm with dynamical interpretations that some asteroids could have migrated inward with the planet to its current orbit at $0.045 \mathrm{AU}$, and that the Yarkovsky effect is ineffective at eliminating objects of $>1 \mathrm{~m}$ in size. However, using numerical models of collisional evolution we find that, due to high relative speeds in this confined Trojan environment, collisions destroy the vast majority of the asteroids in $<10$ Myr. Our modeling indicates that the best candidates to search for exoTrojan swarms in 1:1 mean resonance orbits with "hot Jupiters" are young systems (ages of about 1 Myr or less). Years of Kepler satellite monitoring of such a system could detect an asteroid swarm with a predicted transit depth of $3 \times 10^{-7}$.
\end{abstract}

Key words: minor planets, asteroids: general - stars: individual (HD 209458)

Online-only material: color figures

\section{INTRODUCTION}

Currently, there are slightly over 440 known exoplanets (The Extrasolar Planets Encyclopaedia; http://exoplanet.edu). About 60 of these have orbital planes aligned with our line of sight so that the planets transit their parent stars causing small dips in brightness repeating at the planet's orbital period. Most of the known exoplanetary systems have gas giant planets in small, very short-period orbits, dubbed "hot Jupiters" due to their large sizes and proximities to their parent stars. Dynamical models (Laughlin \& Chambers 2002; Thommes 2005; Cresswell \& Nelson 2006; Lyra et al. 2009) predict that some of these hot Jupiters may be accompanied in their orbits by swarms of Trojan asteroids at the L4 and L5 Lagrangian points, like those in resonance with Jupiter in our own solar system (Stacey $\&$ Connors 2007). The detection and characterization of Trojan asteroids in an exoplanetary system would provide important constraints on the dynamical evolution of giant exoplanets.

Only a few years ago, the prospect of being sensitive to even a lunar mass of asteroids around another star would have seemed like science fiction. The new detection limits made possible by space-based photometry missions like the Microvariability and Oscillations of Stars (MOST), CoRoT, and Kepler mean for the first time that planetary scientists can seriously explore limits on the formation and evolution of possible exoTrojan swarms.

One way of detecting exoplanetary Trojans is through transit timing by combining radial velocity observations and photometric observations of a transiting planet. Through this approach a

\footnotetext{
4 Currently at the Department of Earth and Ocean Sciences, The University of
} British Columbia, 6339 Stores Road, Vancouver, BC V6T 1Z4, Canada.
Trojan swarm can be detected via a time lag between the radial velocity null and the time of the central transit. Using this method, Ford \& Gaudi (2006) ruled out Trojan companions to HD 209458b of total mass greater than $\sim 13 M_{\oplus}$ at a $99.9 \%$ confidence level.

In this paper, we present a more direct and more sensitive approach by searching directly for the transit signal of Trojan swarms with precise space-based photometry. Our target for this study was the HD 209458 system, which contains a hot Jupiter (designated HD 209458b). The planet was discovered in radial velocity measurements by Mazeh et al. (2000) and the transits were first reported by Charbonneau et al. (2000).

HD209458b has a circular orbit of semimajor axis $a=$ $0.045 \mathrm{AU}$ and period $P=3.52474859 \pm 0.00000038$ days (Knutson et al. 2007). The primary HD 209458 is a relatively bright $(V=7.65) \mathrm{G} 0$ star with $T_{\text {eff }}=6000 \pm 50 \mathrm{~K}$, luminosity $L=1.61 L_{\odot}$ (Mazeh et al. 2000), mass $M=1.10 \pm 0.07 M_{\odot}$, and radius $R=1.13 \pm 0.02 R_{\odot}$ (Knutson et al. 2007). Although quite uncertain, Melo et al. (2006) give an estimate of $3 \mathrm{Gyr}$ for the age of the system. HD 209458 is a good starting point for the steps to compare the dynamical state of asteroids in an exoplanetary system with the current state of our solar system: the host star is Sun-like, and it is one of the brightest transiting systems available for study.

It is widely accepted that hot Jupiters formed at orbital distances greater than their current locations and then migrated inward. We explore Trojan survival in such a system. To be visible today, the Trojan populations would have first had to survive a migration from about $5 \mathrm{AU}$ down to about $0.05 \mathrm{AU}$. Once they arrived at this distance, both self-collisions and radiation effects (such as the Yarkovsky effect, which can cause 


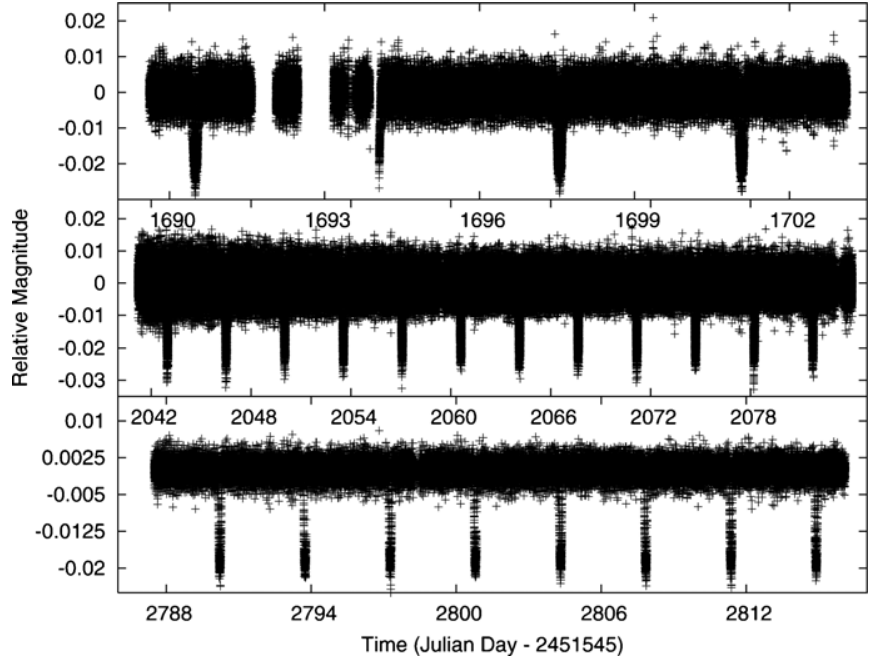

Figure 1. MOST photometry of HD 209458 (from observations in 2004, 2005 and 2007 , respectively) presented as flux variations relative to the mean.

small objects to undergo orbital changes) may be important for these hypothetical "hot Trojans" over time scales comparable to the system's age. We explore these effects through numerical simulations and discuss the results.

This paper is organized as follows. In Section 2, we describe how the photometric data were collected. In Section 3, we describe the data analysis, methods, and results. Section 4 is an estimate of the photometric cross section of the Trojan clouds in the HD 209458 system based on the solar system Trojan size distribution. Section 5 presents numerical calculations regarding dynamical and collisional evolution, as well as the Yarkovsky effect in this system. Finally, Section 6 provides conclusions based on these results.

\section{OBSERVATIONS}

The MOST microsatellite (Walker et al. 2003; Matthews et al. 2004) houses a $15 \mathrm{~cm}$ Rumak-Maksutov telescope feeding a CCD photometer through a single custom broadband filter covering roughly the visible part of the spectrum $(350-700 \mathrm{~nm})$. MOST was launched in 2003 June into an $820 \mathrm{~km}$ high circular Sun-synchronous polar orbit with a period of approximately 101.4 minutes. From this vantage point, it can monitor stars which lie in a roughly equatorial band (continuous viewing zone or $\mathrm{CVZ}$ ) about $54^{\circ}$ wide for up to two months without interruption. Photometry of very bright stars (visual magnitudes $V<6)$ is obtained in Fabry Imaging mode in which a Fabry microlens projects an extended image of the telescope pupil illuminated by the target starlight to achieve the highest precision (Matthews et al. 2004; Guenther et al. 2008). Fainter stars (down to about $V \sim 12$ ) can be observed in direct imaging mode, where defocused images of stars are monitored in subrasters on the CCD (Rowe et al. 2006), similar to groundbased CCD photometry.

MOST monitored the transiting exoplanet system HD 209458 nearly continuously for 13.5 days in 2004 August (a trial run), 42.9 days in 2005 August to September, and 28.6 days in 2007 August to September. These data were used for several purposes: to measure the eclipse (and hence, albedo) of the "hot Jupiter" (Rowe et al. 2006, 2008); to perform transit timing of the known exoplanet to search for lower-mass planets in the system (Miller-Ricci et al. 2008); and to search for planets in the system approaching Earth-size through sensitive transit searches (Croll et al. 2007).

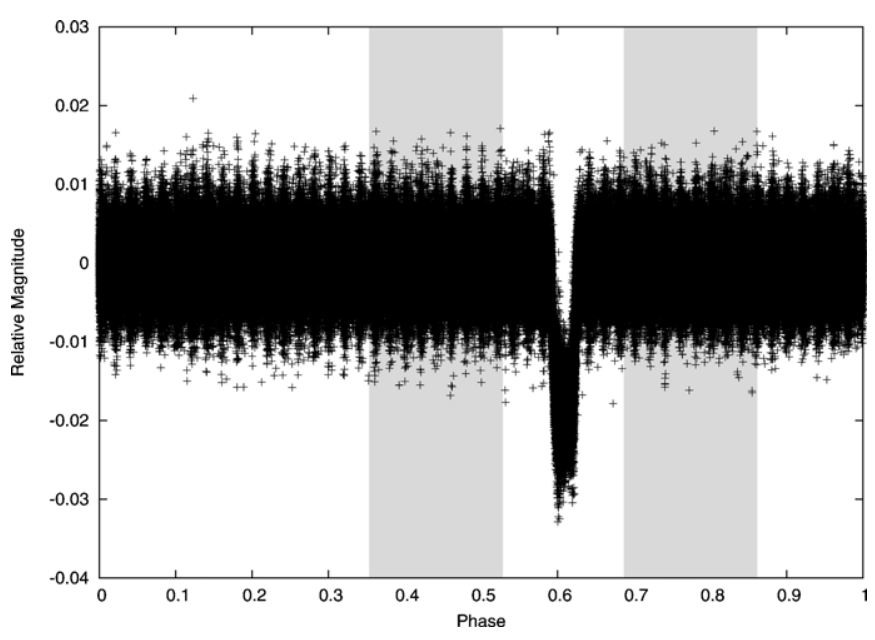

Figure 2. Phase diagram of MOST photometry of HD 209458. Phase is judged relative to the start of the time series, folding at the known planetary orbital period. The planetary transit is obvious. The shaded areas represent sections of the phase diagram where dips in the light curve due to Trojan transits would be expected.

HD 209458 was observed in direct imaging mode with exposures of $1.5 \mathrm{~s}$ sampled every $10 \mathrm{~s}$ (Rowe et al. 2008). The point-to-point precision for these observations ranges from as low as $3 \mathrm{mmag}$ to as high as $20 \mathrm{mmag}$, depending on the level of stray light scattered into the instrument (Miller-Ricci et al. 2008). We rejected exposures with high cosmic-ray fluxes that occur when MOST passes through the South Atlantic Anomaly (SAA), as well as data with background illumination values greater than 3000 detector counts due to scattered earthshine modulated at the satellite's orbital period.

The raw and reduced data are available in the MOST Public Data Archive (http://www.astro.ubc.ca/MOST/data/data.html). The time series photometry is presented in Figure 1, and the data phased according to the orbital period ( $P \sim 3.5248 \mathrm{~d}$ ) of the exoplanet HD 209458b is shown in Figure 2.

\section{SEARCHING FOR TROJANS}

Since Trojan asteroids are expected to concentrate around the L4 and L5 Lagrangian points of the HD 209458 star-exoplanet system, they will share an orbit with the exoplanet. The L4 and L5 points themselves transit the star 1/6th of an orbital period behind and ahead of the exoplanet, with the clouds occupying a confined range of angles surrounding these points. It is expected that these clouds will occupy a fairly wide area (at least $\pm 30^{\circ}$ about the L4/L5 point, as in the case of Jupiter's Trojan clouds) and will therefore have a wide signature in the light curve, each occupying $\simeq 0.2$ of the orbital phase. The multitude of small asteroids would block a small fraction of the stellar light during the period in which the cloud occupies the line of sight. Thus, in the simplest case scenario this would look like a boxfunction dip centered on the L4/L5 point extending about $30^{\circ}$ in each direction. More complicated models (covering a larger angular range, or having a Maxwellian profile, for example) are possible, but are not currently justified by the non-detection from the present data set.

We first examined in more detail the particular phases in the phase diagram shown in Figure 2 where Trojan swarm transits are expected. The exoplanet transits and other obvious outliers were excised from the light curve before our analysis. As a first step, we divided the phased data into six non-overlapping bins, 


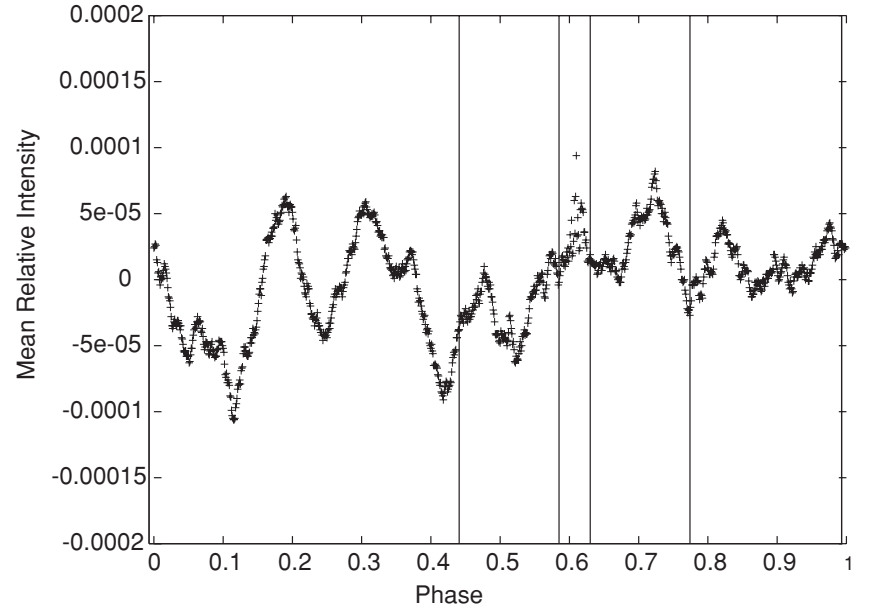

Figure 3. Phase diagram of MOST photometry of HD 209458, binned in overlapping bins of width 0.05 of the orbital cycle, where the bin centers are spaced by only 0.001 cycle. Because of the use of a running average, the noise properties of the signal are masked here; however, if there was a broad $(\simeq 0.2$ in phase) signal due to a transit, it would be apparent in the figure. Orbital phases corresponding to the exoplanet transit (near 0.6) and the transit of the precise L4 and L5 points are indicated by vertical lines.

ensuring that the centers of two of those bins would correspond to the expected center phases of the two possible Trojan swarms. We calculated the mean relative flux value of each of the six bins. This accentuates any possible Trojan signals, as well as having the highest practical signal-to-noise ratio. This exercise, however, did not reveal a significant dip in the binned phase diagram at either the L4 or L5 points.

We then generated running means of the phased data with the width of the phase bins and the bin shift interval as input parameters. A representative result is shown in Figure 3, where the phase bin width is 0.05 cycle and the sampling interval is 0.001 cycle. Since in this case, $96 \%$ of the data in adjacent bins are the same, the resulting means are highly correlated. Figure 3 shows that there are local minima near both Trojan points; however, they are not unique, nor is their extent wide enough to signal Trojan transits.

We tested our sensitivity to Trojan transits in the data by inserting synthetic transits of known duration and depth. A Trojan swarm may have an irregular spatial distribution and hence a more complex transit signature, but for simplicity, we inserted transits of uniform depth $\pm 30^{\circ}$ in size, and decided if we could detect these transits in a diagram like Figure 3 . These tests indicated that our detection limit for Trojan transit depths corresponds to a relative magnitude drop of order $10^{-4}$. We then tackled our detection limits in a more quantitative way via cross-correlation. The time series data were split into a first half and second half of our available time series. The two halves were phased and binned in non-overlapping bins to ensure that there was only one flux value for each phase value. The two sets were also filtered with a low-pass digital Butterworth filter to eliminate any excess ringing in the data that could potentially hide Trojan signals. Then the two data halves were crosscorrelated with each other. Figure 4(a) shows the two filtered data halves, while Figure 4(b) shows the cross-correlation. The mean parametric confidence limits were calculated using the MATLAB code "xcorrc" (Saar \& Manga 2004). Confidence limits are obtained by performing the cross-correlation in the Fourier domain. The code takes the discrete Fourier transform of both data sets, complex conjugates one of them, and then to
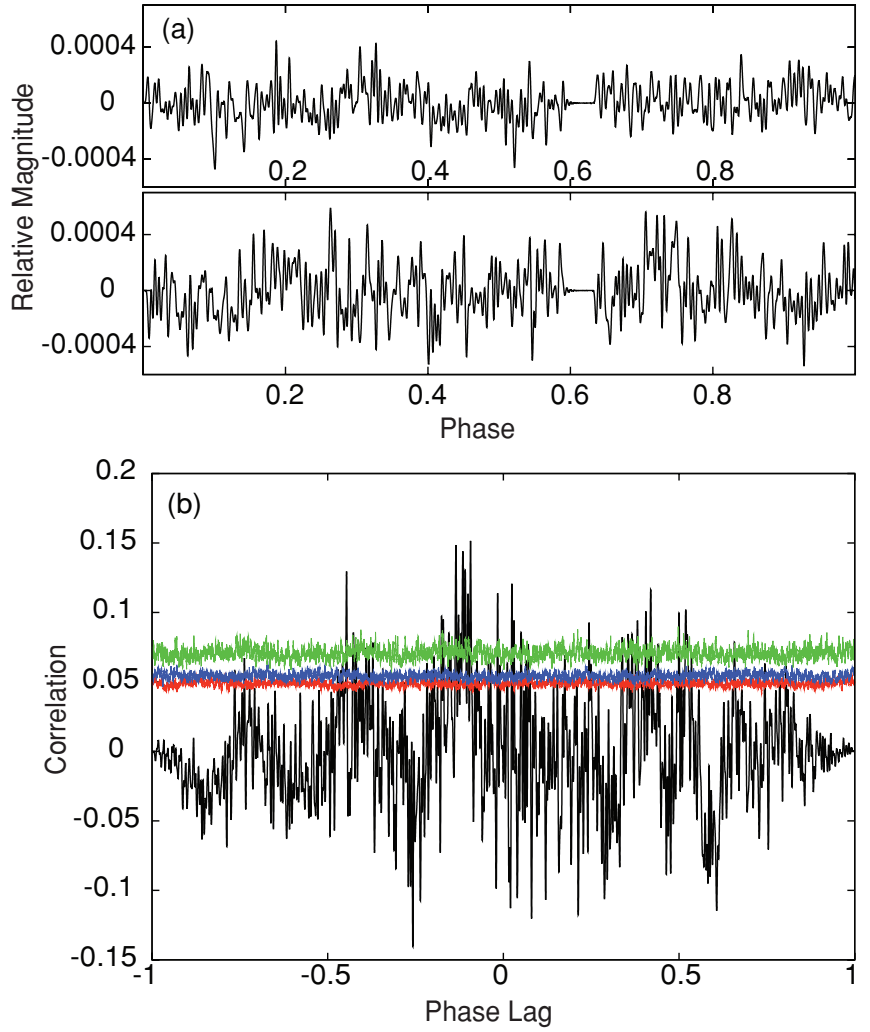

Figure 4. (a) The two data halves before cross-correlation filtered using a normalized cutoff frequency of 0.02 . (b) Cross-correlation of one half of the phased and filtered photometry with the other half. The red, blue, and green curves set the $92 \%$, the $95 \%$, and the $99 \%$ confidence limits, respectively, using 1000 repetitions. A real signal should have $>1 \%$ of the bins centered around zero lag above the $99 \%$ confidence level; no such signal is present.

(A color version of this figure is available in the online journal.)

find the confidence intervals, it introduces chance by randomly replacing the phase values of this second data set. This is then multiplied by the first data set. This procedure is repeated $n$ times, thereby creating a distribution of cross-correlations for each lag value. For example, to set the $95 \%$ confidence interval, xcorrc finds at what level for each lag $95 \%$ of the correlations are below that level (i.e., the level beyond which only $5 \%$ of the peaks are greater strictly by chance).

Any real signal at constant phase with the planet's orbit should have a strong self-correlation at zero relative cross-correlation lag. In addition, if the phases around both L4 and L5 were to generate dimming, there should also be (weaker) crosscorrelation centered close to phase lags of +0.33 and -0.33 (we will illustrate this with artificial signals below). No feature is present in Figure 4 at a correlation lag of zero, nor are broad features centered on +0.33 and -0.33 visible. The weak signal at a phase lag of -0.1 is not significantly stronger than what we expect due to random chance and cannot be a Trojan signal in any case.

We repeated the cross-correlation analysis with artificial transits of known depth inserted into the MOST photometry. The artificial transits were again simple box functions of widths $\pm 30^{\circ}$ centered on the L4 and L5 points. By varying the depth of the artificial transits and calculating the $92 \%, 95 \%$, and $99 \%$ confidence intervals, we estimate the effective Trojan detection limit of our data.

To set a number for our detection limit, we calculated what percentage of the correlation peaks in the original data and 

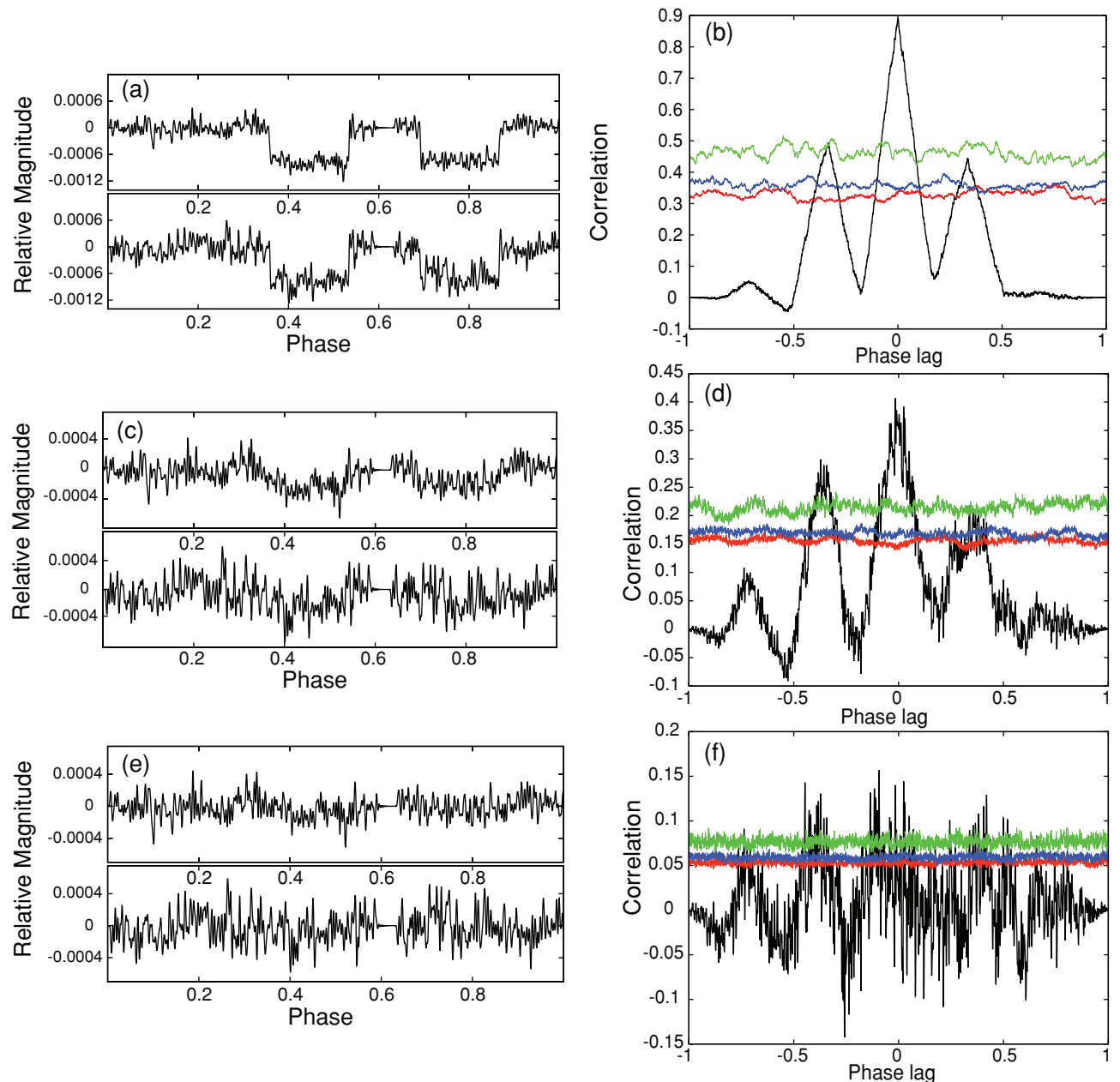

Figure 5. Results from cross-correlation analysis of data with synthetic Trojan transits. The panels on the left-hand side show the two data halves (with artificial Trojan transits) that correspond to the cross-correlations shown in black on the right-hand side. Panels on the right also show the $92 \%, 95 \%$, and $99 \%$ confidence intervals in red, blue, and green, respectively. In panel (a) the artificial Trojan transit depth is $8 \times 10^{-4}$, which shows up as a clear Trojan signal in the cross-correlation (panel b). Panel (c) has artificial transit depths of $2 \times 10^{-4}$. Its corresponding cross-correlation (panel d) shows peaks above the confidence intervals still clearly centered on the lags of $-1 / 3$ and $1 / 3$. Panels (e) and (f) show the data and the cross-correlations, respectively, for a Trojan transit depth of $5 \times 10^{-5}$. In panel (f), the Trojan transits are not clearly discernible.

(A color version of this figure is available in the online journal.)

each synthetic transit scenario were above the $99 \%$ confidence line. For each cross-correlation only $\sim 1 \%$ of the coefficients should be above the $99 \%$ strictly by chance; if this percentage is significantly higher, we can conclude that we are detecting genuine signal in the data. In addition, for it to be a Trojan signal we also require that a strong signal exists near zero phase, and possibly that there are peaks confined around the lags where the Trojans are expected. (The Trojans are expected at lags of approximately $+1 / 3$ and $-1 / 3$, since the L4 and L5 points are $1 / 3$ in phase from each other; these peaks occur when lagged data points at $\mathrm{L} 4$ line up with points at $\mathrm{L} 5$, and vice versa).

Figure 5 shows a few sample cross-correlation results for three artificial Trojan transit tests. For an artificial transit of depth $8 \times 10^{-4}$ (Figures 5(a) and (b)), we recover very strong correlation at zero phase lag and marginally significant signals peaking at the expected \pm 0.33 lags. If only one Trojan point were populated, the side lobes would disappear but the strong zero-lag signal would remain. An acceptable signal from artificial Trojans with dips of $2 \times 10^{-4}$ is shown in Figures 5(c) and (d). The zero-phase correlation is strong but, as expected, the \pm 0.33 peaks have become less pronounced for shallower depths of transit (in fact, one of them is only barely significant). For a transit depth of $5 \times 10^{-5}$ (Figures 5(e) and (f)), only very weak correlations remain, due to the much reduced signal to noise, and we judge this a non-detection since the zero-phase signal is no stronger than the -0.33 feature. From this analysis, we conclude that our detection limit is $\simeq 10^{-4}$, the same as from our eyeball analysis of the data.

\section{TROJAN SIZE DISTRIBUTION—SOLAR SYSTEM MODEL}

To estimate the flux reduction due to possible Trojan clouds in the HD 209458 system, the known properties of Jupiter's Trojan swarms in our solar system were applied to the HD 209458 system. In this way, we estimate the expected transit depth if the Trojans in the HD 209458 system had the same size distribution.

We first calculated the cross-sectional area of the Trojan clouds given this size distribution, as well as the mass of the swarms, and from there the transit depth was established. 


\subsection{Cross-sectional Area of a Trojan Cloud}

We estimated the cross-sectional area of a single Trojan cloud by integrating the relation

$$
d a=\pi r^{2} n d r
$$

where the differential size distribution $n d r$ measures with one power law for radii in the range $2.2 \mathrm{~km} \leqslant r \leqslant 42 \mathrm{~km}$ and another for $r \geqslant 42 \mathrm{~km}$ (Jewitt et al. 2000). For radii smaller than $r \leqslant 2.2 \mathrm{~km}$, we calculated a differential size distribution by matching the Jewitt et al. result with that of Yoshida \& Nakamura (2005) (see Figure 6). For $r \geqslant 42 \mathrm{~km}$, Jewitt et al. (2000) give

$$
n_{r>42}(r) d r=3.5 \times 10^{9}\left(\frac{1 \mathrm{~km}}{r}\right)^{5.5 \pm 0.9} d r .
$$

In the range $2.2 \mathrm{~km} \leqslant r \leqslant 42 \mathrm{~km}$, Jewitt et al. give

$$
n_{42>r>2.2}(r) d r=1.5 \times 10^{6}\left(\frac{1 \mathrm{~km}}{r}\right)^{3.0 \pm 0.3} d r
$$

which matches at the $42 \mathrm{~km}$ break. Yoshida \& Nakamura (2005) find a cumulative power-law slope of 1.3 for Trojans with radii between $1 \mathrm{~km} \leqslant r \leqslant 2.5 \mathrm{~km}$. Here, we force a match at $r=2.2 \mathrm{~km}$, adopting $n_{42>r>2.2} d r=n_{2.2>r} d r=1.41 \times 10^{5} d r$, from which we calculate the third power-law $n_{r>2.2} d r$ as

$$
n_{2.2>r}(r) d r=c \cdot\left(\frac{1 \mathrm{~km}}{r}\right)^{2.3 \pm 0.1} d r
$$

where $c=8.65 \times 10^{5} \mathrm{~km}^{-1}$. To find the total cross-sectional area of the Trojans, we substitute these distributions into Equation (1) and integrate to find the total cross-sectional area $a_{\text {trojans }}=a_{r>42}+a_{42>r>2.2}+a_{2.2>r}$. Using the best estimates for the slopes, the cross sections from the three distributions are

$$
a_{r>42}=3.5 \times 10^{9} \cdot \pi \int_{42}^{\infty}\left(\frac{1 \mathrm{~km}}{r}\right)^{5.5} r^{2} d r \approx 1 \times 10^{6} \mathrm{~km}^{2},
$$

$a_{42>r>2.2}=1.5 \times 10^{6} \cdot \pi \int_{2.2}^{42}\left(\frac{1 \mathrm{~km}}{r}\right)^{3} r^{2} d r \approx 1.4 \times 10^{7} \mathrm{~km}^{2}$,

and

$$
a_{2.2>r}=8.65 \times 10^{5} \cdot \pi \int_{0}^{2.2}\left(\frac{1 \mathrm{~km}}{r}\right)^{2.3} r^{2} d r \approx 7 \times 10^{6} \mathrm{~km}^{2} \text {. }
$$

While the asteroids smaller than $2.2 \mathrm{~km}$ do not contribute significantly to the mass of the Trojan clouds (see below), they do contribute significantly to the cross-sectional area and therefore the depth of transit. Our estimate of the total crosssectional area of a Trojan cloud is $a_{\text {trojans }} \approx 2 \times 10^{7} \mathrm{~km}^{2}$. Although it is conceivable that our Trojan cloud's optical depth could be governed by a large population of $r \ll 1 \mathrm{~km}$ particles which are currently unobservable, given the lack of evidence for this hypothesis we shall use $2 \times 10^{7} \mathrm{~km}^{2}$ as our estimate of the total Trojan cross section. Beyond this concern, the dominant source of uncertainty is the power-law indices in the size distribution, producing a variation of a factor of a few in the total cross section if we instead adopted a size distribution similar to Fernandez et al. (2009).

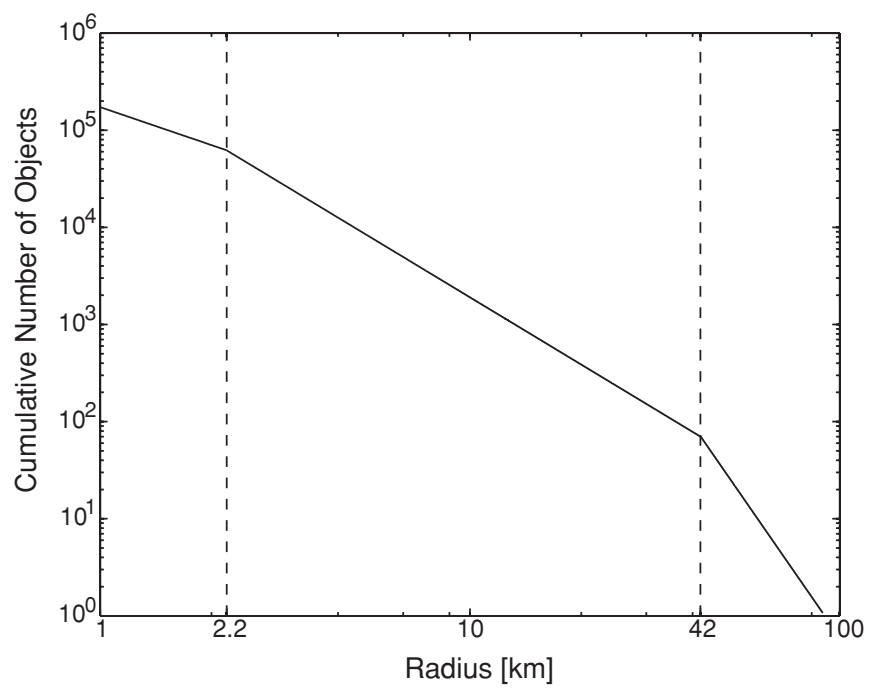

Figure 6. Cumulative size distribution for solar system asteroids based on Jewitt et al. (2000) and Yoshida \& Nakamura (2005). The breaks in the distribution occur near an asteroid radius of $2.2 \mathrm{~km}$ and $42 \mathrm{~km}$.

\subsection{Mass of Trojan Cloud: Our Solar System's}

We calculate the total mass of Trojans by integrating the three differential size distributions and assuming a mean asteroid density of $\bar{\rho}=2000 \mathrm{~kg} \mathrm{~m}^{-3}$. In this way, Jewitt et al. (2000) estimated the total mass of solar system Trojans with $r>2.2 \mathrm{~km}$ as $M_{T} \approx 5 \times 10^{20} \mathrm{~kg}$. We extend this result by including the third differential size distribution $n_{2.2>r} d r$. The total mass of Trojans is then

$$
\begin{aligned}
M_{T}= & \int_{0}^{2.2} \frac{4}{3} \pi \bar{\rho} r^{3} n_{2.2>r} d r+\int_{2.2}^{42} \frac{4}{3} \pi \bar{\rho} r^{3} n_{42>r>2.2} d r \\
& +\int_{42}^{\infty} \frac{4}{3} \pi \bar{\rho} r^{3} n_{r>42} d r
\end{aligned}
$$

This yields $M_{T} \sim 5.9 \times 10^{20} \mathrm{~kg}$, containing (as expected) negligible additional mass in the distribution tail with $r<$ $2.2 \mathrm{~km}$. Expressed in lunar masses $\left(M_{\text {Moon }} \sim 7.36 \times 10^{22} \mathrm{~kg}\right.$ ), this is about $0.008 M_{\text {Moon }}$. This mass estimate should be halved if the measured density of $\rho \sim 1000 \mathrm{~kg} \mathrm{~m}^{-3}$ for the Trojan Patroclus is common (Marchis et al. 2006).

\subsection{Depth of Transit}

To estimate the drop in measured flux of the star due to a population of Trojans as described above, we assume that Trojans with that size distribution are orbiting the star HD 209458a surrounding the L4 and L5 points of HD 209458b. Given our null detection, we believe only order-of-magnitude estimates are warranted at this time. We take the star's radius to be $R_{\text {star }}=1.13 R_{\text {Sun }}=7.85 \times 10^{5} \mathrm{~km}$, and assume Trojans to be evenly distributed around the L4 and L5 points. If all the L4 Trojans, or all the L5 Trojans (whose populations we take to be equal and thus half the total), were in front of the star at the same time, the drop in light output would be

$$
\frac{\Delta I}{I}=\frac{a_{\text {trojans }} / 2}{a_{\text {star }}} \sim 6 \times 10^{-6},
$$

where we are neglecting the effects of limb darkening. However, we need to account for the fact that the longitudinal extent of the Trojan cloud at either of the Lagrangian points will most likely 
be larger than the angular size of the star. Very roughly, the longitudinal extent of Jupiter's Trojans around one Lagrangian point is about $\pm 30^{\circ}$. The projected stellar diameter, expressed in terms of the exoplanet's orbit, is the fraction of that orbit covered by the planet's transit, which is 0.035 in phase $\left(13^{\circ}\right)$. This means that a Trojan cloud like that of Jupiter in the HD 209458 system has only about a fifth of its Trojans in front of the star during transit. Therefore, we divided our drop in magnitude by a factor of 5 giving $\frac{\Delta I}{I} \sim 1.2 \times 10^{-6}$.

Another issue that may affect the star's brightness is that Jupiter's Trojans have a large dispersion above and below the ecliptic plane. From Yoshida \& Nakamura (2005), the average Trojan inclination is $10^{\circ}$, and we take the putative exoplanet Trojan system to be $\sim 30^{\circ}$ (where Section 5.1 motivates the factor of 3 increase). Knowing that the Trojan semimajor axis is $a=0.045 \mathrm{AU}$ and that the star's radius is $R_{\text {star }}=0.0052 \mathrm{AU}$, the "height" of the Trojan cloud is $\sim 5$ stellar diameters above and below the orbital plane. Thus, we divide our $\frac{\Delta I}{I}$ by 5 , although this effect will not occur if the HD 209458b Trojan orbits have inclinations significantly $<30^{\circ}$. Our final estimate for the drop in the star's flux due to the transit of a Jovian-type Trojan cloud is $\frac{\Delta I}{I} \sim 3 \times 10^{-7}$. To detect such a transit requires a photometric precision of order $3 \times 10^{-7}$ assuming the Trojans obey the solar system size and angular distributions.

\subsection{Exotrojan Mass}

Based on the above calculations, we estimate the mass of the hypothetical Trojan cloud in the HD 209458 system that would be required for MOST to have a detection. Since our detection limit for a Trojan transit is a drop in the light output of $1 \times 10^{-4}$, we would not be able to detect the $3 \times 10^{-7}$ drop caused by a Trojan population of the size of Jupiter's Trojan clouds. However, for

$$
\left.\frac{\Delta I}{I}\right|_{\text {limit }}=1 \times 10^{-4}=\frac{a_{\text {trojans }}}{a_{\text {star }}},
$$

so we find that the Trojan cross-sectional area is $a_{\text {trojan }} \sim$ $2 \times 10^{8} \mathrm{~km}^{2}$, which is a factor of $\sim 100$ times the optical depth of Jupiter's Trojan cloud. Therefore, MOST could detect a Trojan cloud in front of HD 209458a if the number of asteroids in each size bin (assuming the size distribution of Jupiter's Trojans) were increased by a factor of 100 . This would increase the mass of the entire cloud by this factor, thus bringing the minimum exotrojan mass that we could detect to $\sim 1$ lunar mass.

\section{DYNAMICAL EFFECTS}

In this section, we discuss the relationship between the upper limit on the HD 209458b Trojan population given by our nondetection and what one might expect to exist in the exoplanet system. In order to have some concreteness (in what is an otherwise large and unconstrained parameter space), we will mostly concentrate on the scenario in which HD 209458b forms at $\sim 5 \mathrm{AU}$ from its star along with a Trojan population equivalent to some multiple of Jupiter's current population, after which it migrates to its current position, and then the Trojans and planet remain at the current stellar distance.

The efficient mechanisms for planet migration, in which the planet couples to the gas disk, would require that the planet migrated to its current position within the first $\sim 3-10 \mathrm{Myr}$ of the system's lifetime, before the circumplanetary gas was dissipated. Since HD 209458 is $\sim 3$ Gyr old, the time that the system has been at its current orbital distance is $2-3$ orders of magnitude longer than the migration phase.

\subsection{Migration Phase}

As the planet migrated in toward the star the 1:1 resonant Trojans also spiraled inward, remaining trapped in the resonance although their libration amplitudes grew. (The libration amplitude $A$ measures the total amplitude of the angular variation of a given particle away from the Trojan point in the reference frame co-rotating with the planet). We have conducted straightforward numerical simulations to confirm the result of Fleming \& Hamilton (2000) that the libration amplitude slowly grows as the planet migrates inward according to

$$
A_{f}=\left(\frac{a_{f}}{a_{i}}\right)^{-1 / 4} A_{i},
$$

where $a$ is the semimajor axis of the planet's orbit and subscripts $i$ and $f$ indicate the initial and final values of the variables (before and after migration). Fleming \& Hamilton (2000) show that the eccentricity and inclination of the Trojan orbits grow by the same factor. Assuming HD 209458b began about 5 AU from the star, its current semimajor axis results in Trojan libration amplitudes growing by a factor of $\sim 3.3$ while migrating, which is confirmed in our numerical simulations. This results in a large fraction of the Trojan phase space having libration amplitudes that grow beyond the maximum possible stable value (of about $130^{\circ}$ ) and leave the resonance, after which they will interact with the planet and be accreted or ejected. Using a uniformly filled initial Trojan phase space results in $\sim 10 \%$ of the Trojans (those with initial libration amplitudes $<35^{\circ}$ ) surviving migration, where the resultant stable co-orbitals have libration amplitudes that have grown to fill the stable libration region. Given what follows below, improved precision on these estimates is not currently warranted.

\subsection{Collisional Evolution}

A small-body population, like our asteroid belt, will have its population and size distribution evolve collisionally if the spatial density is sufficiently high and relative speeds are large enough that collisions are frequent and cause net erosion. In the case of a hypothetical HD 209458 b cloud, collisions could be occurring in the pre-migration phase, during the planetary migration, and then in the post-migration period. Since the final stage is both much longer and has the highest relative speeds and spatial density (since the Trojans occupy much less volume when they surround the Trojan point at $0.045 \mathrm{AU}$ than at $\sim 5 \mathrm{AU}$ ), we will neglect the collisional evolution except during the post-migration phase. This collisional evolution will result in collisional fragmentation of Trojans, which will move mass from large objects in a size distribution down to smaller diameters; when collisions produce particles small enough that radiation-pressure effects become important, they are quickly eliminated from the system. Collisions will thus grind down the total mass of the system on some time scale. In our solar system, the internal collisional cross section is low enough that the main asteroid belt's mass has not significantly been reduced by grinding over the last 4 billion years (Bottke et al. 2005).

We posed the questions: if a Trojan cloud survives migration down to $0.045 \mathrm{AU}$, how would it evolve? If collisions are important, can we postulate an increased initial mass in order to obtain enough Trojans today to have an observable 


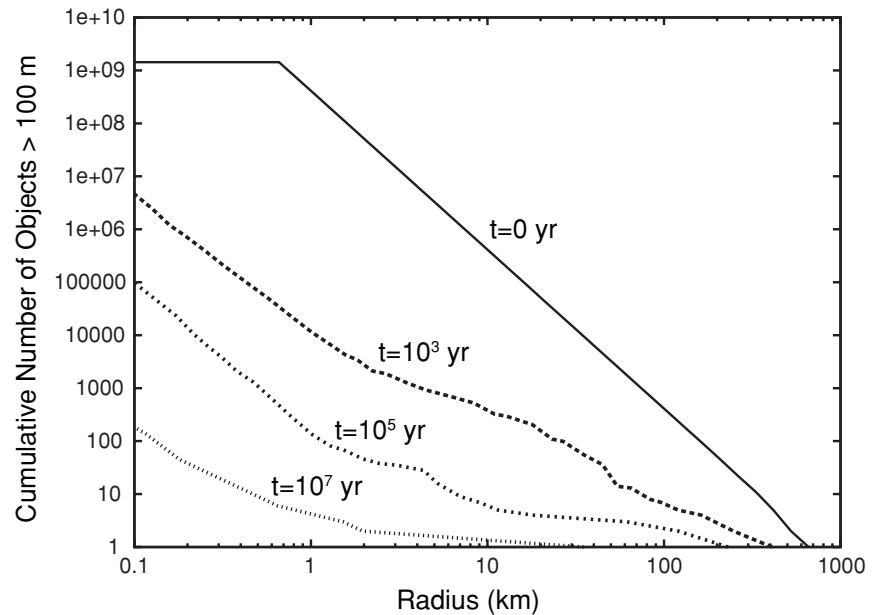

Figure 7. Results from dynamic collisional simulation for initial 1 lunar mass of Trojans. Here, the cumulative number of objects greater than $100 \mathrm{~m}$ is plotted as a function of object radius for various time steps in the simulation. By $10 \mathrm{Myr}$ there is almost nothing left of the original size distribution (the program does not keep track of objects less than $\sim 100 \mathrm{~m}$ ). Thus, from this plot we can see that the cloud grinds itself down to meter-sized objects within a few million years.

signal? Recall that Jupiter's Trojan cloud has a mass $\sim 0.01$ lunar masses, so we will use order-of-magnitude multiples of this for hypothetical populations. Trojan clouds with initial orbital eccentricities and inclinations similar to our solar system Trojans were used, which determines the collision speeds to be $\sim v_{\text {kep }} \sqrt{e^{2}+i^{2}} \sim 65 \mathrm{~km} \mathrm{~s}^{-1}$, where $v_{\text {kep }}$ is mean orbital speed of the Trojans. If migration results in even higher typical $e$ 's and $i$ 's, then these speeds would grow by a factor of 3-4. This enormous mutual velocity means that collisions are very destructive when compared to those occurring in our asteroid belt, where mean speeds are only about $5 \mathrm{~km} \mathrm{~s}^{-1}$ (Bottke et al. 1994). Although this mutual speed could be decreased if the mutual $e$ 's and $i$ 's were dropped, the mutual collisional cross section increases under this change because the volume occupied by the cloud decreases.

The Trojan swarm was evolved collisionally using an algorithm very similar to that described in Morbidelli et al. (2009), which keeps track of the evolving size distribution of the collisional swarm. Figure 7 shows the evolution of the size distribution between 0.1 and $1000 \mathrm{~km}$ at various times in the evolution for an initial 1 lunar-mass population, with an initial differential power-law size index of -3 . The population is rapidly decimated by mutual collisions, which by 10 Myr leaves almost all the mass in a single $35 \mathrm{~km}$ (radius) object while reducing the number of $1 \mathrm{~km}$ objects (where most of the cross section is) by more than 8 orders of magnitude. Thus, even if an initial Trojan swarm of 1000 times that of Jupiter started at 5 AU and then arrived at $0.045 \mathrm{AU}$ in a migration that reduced it by an order of magnitude, in only $10 \mathrm{Myr}$ the light-blocking cross section is reduced by more than a factor of a million, making detection today many orders of magnitude below current or projected flux limits for HD 209458b. A few million years into the simulations, the total Trojan cross-sectional area is $\sim 10^{-8}$ that of the star, and continues to decrease.

Neither increasing nor decreasing the mass of the cloud that arrives at 0.045 AU helps. Additional simulations with 1, 2, or 3 orders of magnitude more or less initial mass arrive at about the same final state after $10 \mathrm{Myr}$ of collisions, due to the simple fact that the collision rate is proportional to the

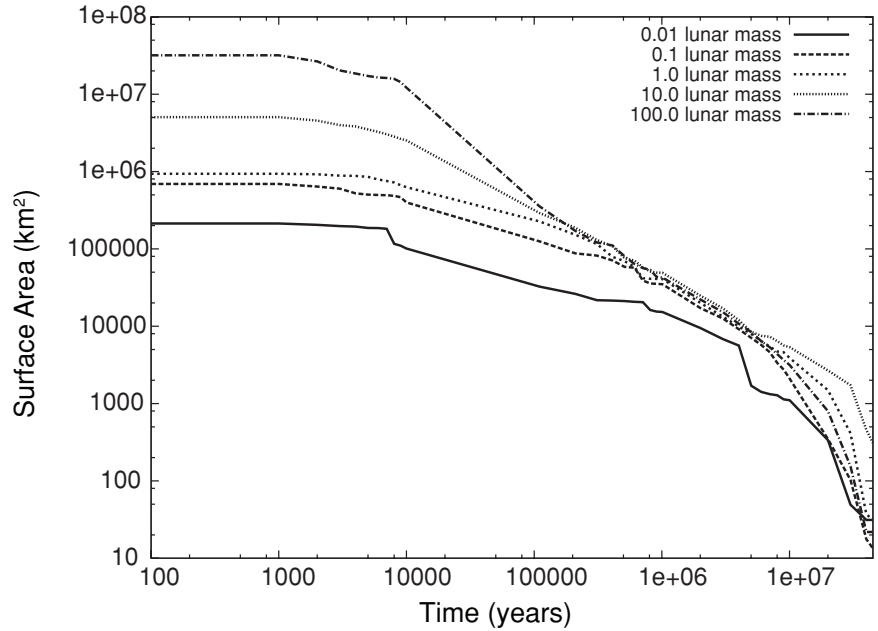

Figure 8. Surface area of Trojan cloud as a function of time from simulation for various initial cloud masses. The initial total mass of the cloud (in lunar masses) is indicated beside each distribution. The total surface area (from objects with radii $>50 \mathrm{~m}$ ) of the cloud decreases with time independent of the initial starting mass. After $\sim 1 \mathrm{Myr}$, the surface areas for the different initial masses are the same within a factor of 3 of each other.

number of objects. In fact, the total amount of mass after $10 \mathrm{Myr}$ is comparable to the $0.01 \mathrm{Myr}$ state (see Figure 8) since the system grinds itself down to the point where the collisions "turn off" because the remaining objects have so little mutual cross section.

Experiments with -4 power-law indices gave similar results. Our conclusion is that, unless the collisional modeling is wrong by many orders of magnitude, Trojan clouds with detectably large cross-sectional area will not survive for even a million years after the planet migrates to hot Jupiter distances. Hiding the mass in a smaller number of large bodies would prevent collisions from destroying the mass, but these bodies have such small cross-section/mass ratios that such a population would have negligible optical depths and cannot be detected via light curve technology in the foreseeable future.

\subsection{Emptying the Trojan Points}

The Trojan population which initially arrives with the planet at $0.045 \mathrm{AU}$ at the end of the migration phase is initially modified by collisions. The collisional cascade fragments larger bodies and populates the smaller-diameter bins, whose bodies in turn are destroyed by collisions among smaller particles. In our asteroid belt this process stops when particles are ground down to submillimeter size at which point radiation forces eliminate them, or when bodies of 0.01-1 km scale have their semimajor axes slightly modified by Yarkovsky drift which moves them into a resonance at which point they can rapidly leave the belt (reviewed in Bottke et al. 2006). The migration rates induced by these processes and migration direction (toward or away from the star) depends on the particle's size, spin rate, obliquity, and the thermal properties of the surface.

In the case of exoplanet Trojans, these forces do not cause a gradual monotonic semimajor axis drift (which would be $\sim$ $\pm 1 \mathrm{~mm} \mathrm{~s}^{-1}$ for a $1 \mathrm{~m}$ diameter rocky object). A straightforward analysis of the circular restricted three-body problem shows that the additional constant acceleration, which would produce a slow semimajor axis change outside the resonance, instead causes a tiny change $\left(<1\right.$ part in $\left.10^{4}\right)$ in the positions of the Lagrange points. This effect is analogous to how a small 
damping force added to a driven harmonic oscillator only produces a phase shift.

This shift in the location of the libration centers is not in itself a source of instability, and thus the radiation damping would not cause the particles to leave the Trojan points. However, because the libration center moves if the object suffers a collision which changes its spin rate and direction, the libration amplitude will random walk toward larger values and result in the eventual destabilization of the Trojan; we estimate this time scale to be $\sim 1$ Gyr for metre-scale Trojans of HD 209458b. We showed above that the rapid collisional grinding efficiently transfers mass from larger objects $(>1 \mathrm{~km})$ down to the regime where radiation effects can then lead them to be destabilized and pulled down to the star. In particular, once free of the resonance, metrescale objects at these distances will spiral into the star from Poynting-Robertson drag in only $2 \mathrm{Myr}$, with the time scale proportional to the object size (Gladman \& Coffey 2009). As a result of these processes, there may be a period during the system evolution where a large amount of mass has moved into the diameter region just above where radiation-pressure effects are efficient at eliminating small particles. This will be the size regime with the best optical depth to mass ratio, and it is possible that a nearly opaque cloud in one or both Trojan points could be temporarily produced. Such a cloud would have a very strong photometric signature (essentially blocking the stellar light for the portion of the disk that is eclipsed, for the portion of rotational phase that it is in front of the star). Our collisional simulations above indicate that this would be a brief (certainly $<1$ Myr) phase that would most likely occur in a very young system during the grinding phase before the mass is eliminated. (In fact it is possible that it might occur during the migration phase itself). This could also occur in older systems if a large body manages to survive in a Trojan point but is then broken up, beginning a similar rapid collisional cascade at that time.

\section{CONCLUSIONS}

In this paper, photometric data on HD 209458 from the MOST space telescope were analyzed with the intent of finding Trojan asteroids swarms in 1:1 mean motion resonance with the known planet HD 209458b. Using cross-correlation techniques on data with synthetic Trojan cloud transits, we were able to set an upper limit on the Trojan transit depth of $1 \times 10^{-4}$, which corresponds to an upper limit in asteroid swarm mass to $\sim 1$ lunar mass.

We then assessed the dynamical effects on such a Trojan cloud in the HD 209458 system. The main findings are as follows.

1. During the migration of the Trojan swarm (as they migrate with the planet from $\sim 5 \mathrm{AU}$ to $0.045 \mathrm{AU})$, the libration amplitudes grow by a factor of $\sim 3.3$ leaving $\sim 10 \%$ of the Trojans surviving migration.

2. The collisional evolution of the cloud grinds it down to below $100 \mathrm{~m}$ size objects in $\ll 10$ Myr and reduces its surface area 10,000 fold. Neither increasing nor decreasing the initial mass of the cloud changes the results significantly. After only a few Myr the total cross section remaining in the Trojan swarm (in bodies larger than tens of meters) has dropped to $<10^{-8}$ that of the star. Based on the initial photometric performance of Kepler long cadence data (Jenkins et al. 2010), for a solar-type star of $V \sim 10$ with Trojan asteroid swarms in a 3.5 day orbit, the light curve would reach a sensitivity to transit depth of $3 \times 10^{-7}$.
In a young system, this would be sufficient to detect the exoTrojan swarms based on our model predictions.

3. Radiation forces do not cause a semimajor axis drift of the leftover small objects, only a shift in the location of their libration centers. Further collisions between these small objects could then cause a random walk in their libration amplitudes, eventually destabilizing the Trojans and eliminating them on a time scale of $2 \mathrm{Myr}$.

4. A nearly opaque cloud might result from the collisionally ground asteroid population just before the radiation forces take effect. Such a cloud would have a very strong photometric signature for a brief time period ( $<1 \mathrm{Myr}$ ) due to the dense population of small particles. This would occur during the collisional phase of the cloud evolution, and since this is a fast process, this could only be observed in a very young system $(\ll 100 \mathrm{Myr})$.

Thus, the best chance of detecting Trojan asteroid swarms in another solar system in the foreseeable future would be in very young systems, where either the collisional depletion has not yet reduced the surface area significantly, or where the cloud turned opaque for a short time due to the high density of centimetersized objects before elimination by Poynting-Robertson drag. Detection in an older system would require a recent breakup of a large body that generated a collisional cascade of smaller objects.

Our simulations do rely on the assumption of simple migration of the gas giant planet. Other processes, involving planet-planet interactions and later tidal circularization of the exoplanet's small orbit, could lead to different scenarios of exoTrojan migration and evolution. However, we note that most of the observations of the Rossiter-McLaughlin effect in exoplanet systems, including HD 209458 (Winn et al. 2005), have pointed to only small levels of spin-orbit misalignment in systems with close-in giant planets (Winn 2007; Narita et al. 2009). There is no reason to strongly suspect planet-planet interactions in the case of HD 209458 and other hot Jupiter systems with small, nearly circular orbits.

What about exoplanets in orbits with much larger semimajor axes? For exo-Jupiters with relatively large semimajor axes, the prospects of detecting transits in the existing sample are limited. The Kepler mission will expand the sample and should change that situation in the coming years. Many of the known exoplanets with larger semimajor axes also have high eccentricities. In these systems, there could have been dynamical interactions with other planets earlier in the histories of the systems. We argue that these are not systems where it would be fruitful to search for Trojans in resonant orbits, and for that reason, we did not explore this broader range of parameter space in the numerical simulations presented in this paper.

Our analysis of ultra-precise MOST photometry provides the most sensitive upper limit yet published on the amount of Trojan asteroidal material in an exoplanetary system. This upper limit inspired us to perform numerical simulations to show what Trojan optical depth might be present. By estimating the expected optical depth of an exoplanetary asteroid cloud, we have set meaningful limits on future photometric searches for exoTrojan swarms by missions such as Kepler, and focus attention in particular on very young systems.

R.M., J.M.M., and B.G. acknowledge the support of NSERC. We thank the referee for insightful comments which improved the paper. 


\section{REFERENCES}

Bottke, W. F., Durda, D. D., Nesvorny, D., Jedicke, R., Morbidelli, A., Vokrouhlicky, D., \& Levison, H. F. 2005, Icarus, 179, 63

Bottke, W. F., Nolan, M. C., Kolvoord, R. A., \& Greenberg, R. 1994, Icarus, 107,255

Bottke, W. F., Vokrouhlicky, D., Rubincam, D. P., \& Nesvorny, D. 2006, Ann. Rev. Earth Planet. Sci., 34, 157

Charbonneau, D., Brown, T. M., Latham, D. W., \& Mayor, M. 2000, ApJ, 529, L45

Cresswell, P., \& Nelson, R. P. 2006, A\&A, 450, 833

Croll, B., et al. 2007, ApJ, 658, 1328

Fernandez, Y., Jewitt, D., \& Ziffer, J. 2009, AJ, 138, 240

Fleming, H. J., \& Hamilton, D. P. 2000, Icarus, 148, 479

Ford, E. B., \& Gaudi, B. S. 2006, ApJ, 652, L137

Gladman, B., \& Coffey, J. 2009, Meteorit. Planet. Sci., 44, 285

Guenther, D. B., et al. 2008, Commun. Asteroseismol., 157, 64

Jenkins, J. M., et al. 2010, ApJ, 713, L120

Jewitt, D. C., Trujillo, C. A., \& Luu, J. X. 2000, AJ, 120, 1140

Knutson, H. A., Charbonneau, D., Noyes, R. W., Brown, T. M., \& Gilliland, R. L. 2007, ApJ, 655, 564

Laughlin, G., \& Chambers, J. E. 2002, AJ, 124, 592
Lyra, W., Johansen, A., Klahr, H., \& Piskunov, N. 2009, A\&A, 493, 1125

Marchis, F., et al. 2006, Nature, 439, 565

Matthews, J. M., Kuschnig, R., Guenther, D. B., Walker, G. A. H., Moffat, A. F. J., Rucinski, S. M., Sasselov, D., \& Weiss, W. W. 2004, Nature, 430, 51

Mazeh, T., et al. 2000, ApJ, 532, L55

Melo, C., Santos, N. C., Pont, F., Guillot, T., Israelian, G., Mayor, M., Queloz, D., \& Udry, S. 2006, A\&A, 460, 25

Miller-Ricci, E., et al. 2008, ApJ, 682, 586

Morbidelli, A., Nesvorny, D., Bottke, W. F., \& Levison, H. F. 2009, Icarus, 204, 558

Narita, N., et al. 2009, PASJ, 61, 991

Rowe, J. F., et al. 2006, ApJ, 646, 1241

Rowe, J. F., et al. 2008, ApJ, 689, 1345

Saar, M. O., \& Manga, M. 2004, J. Geophys. Res., 109, B04204

Stacey, R. G., \& Connors, M. 2007, Planet. Space Sci., 56, 358

Thommes, E. W. 2005, ApJ, 626, 1033

Walker, G. A. H., et al. 2003, PASP, 115, 1023

Winn, J. N., et al. 2005, ApJ, 631, 1215

Winn, J. N. 2007, in ASP Conf. Ser. 366, Transiting Extrapolar Planets Workshop, ed. C. Afonso, D. Weldrake, \& Th. Henning (San Francisco, CA: ASP), 170

Yoshida, F., \& Nakamura, T. 2005, AJ, 130, 2900 